\title{
A Computational Model of Autonomic Nervous System for Heart Rate Variability
}

\author{
Sajitha $\mathrm{S}^{1}$, Minimol Balakrishnan², Mini $\mathrm{M} \mathrm{G}^{3}$ \\ ${ }^{1}$ Model Engineering College, Kochi, India
}

\begin{abstract}
Heart Rate Variability (HRV) is the subtle beat to beat changes in heart rate. Autonomic Nervous System (ANS) regulates heart rate by controlling the neurotransmitters, mainly Norepinephrine (NE) and Acetyl choline (Ach) from sympathetic and parasympathetic branches respectively. HRV analysis is a noninvasive tool for assessing the integrity of ANS. HRV changes are observed in the onset of heart disease and in a number of disease conditions like sleep apnea, psychiatric disorders, diabetes, hypertension etc. An understanding of the relationship between kinetics at sympathetic and parasympathetic sites and HRV helps to identify biological changes associated with various autonomic imbalance conditions and hence help in targeted diagnosis and therapy. A computational model of ANS for heart rate regulation is proposed in this study. Fitzhugh Nagumo (FHN) model is used as the successive stage of proposed model to generate a discrete time heart beat interval series. HRV data from a group of healthy individuals having balanced sympathetic and parasympathetic activities were studied. The results were in agreement with parameters derived from model synthesized data for the same autonomic state.
\end{abstract}

\section{Introduction}

Autonomic Nervous System (ANS) innervates almost all vital organs of the body. It has important functions in the body such as control of heart rate, respiratory rate, gastro intestinal motility, pupillary response, urination etc. [1]. Hence integrity of ANS is absolutely necessary for the physical and mental wellbeing of a person. ANS retains a dynamic balance between sympathetic and vagal neural traffic and this is reflected in heart rate variability (HRV). So HRV can indicate how effectively ANS performs its functions in the body.

Heart rate regulation is the major mechanism through which Cardio Vascular System (CVS) meets the need for varying blood demand in the body. HRV changes have been implicated in the early onset of heart disease $[2,3]$, psychiatric disorders like depression [4], schizophrenia
[5], respiratory disorders like Obstructive Sleep Apnea Syndrome(OSAS) [6], and lifestyle diseases like hypertension [7] and diabetes [8]. Nowadays clinicians use HRV measures for diagnosis, assessing the progress of treatment and effects of certain drugs [9]. HRV has been a largely explored area of research for the last two decades; various methods for the analysis of HRV have been developed [10]. A quantitative model of heart rate mechanism seems to be significant in the present scenario; as it can precisely pinpoint the underlying physiological change in disease conditions. A mathematical model of ANS for heart rate regulation is proposed in the present study.

Heart rate regulation is effected as a result of various mechanisms that work at different time scales. The cardiovascular center in the medulla integrates information from the baroreceptors, chemoreceptors and proprioceptors of the heart. In response to this information the relative balance between the sympathetic and vagal neural traffic is adjusted which in turn effects a change in the rate of depolarization of auto rhythmic cells of the heart.

Increased efferent activity in vagal nerves causes enhanced release of Acetyl choline (Ach), which on binding with M2 muscarinic receptors results in a decreased rate of depolarization of heart. Sympathetic activity causes release of Norepinephrine (NE); which on binding with beta adrenergic receptors effects increase in heart rate. At rest both the branches of ANS are active but vagal system dominates [11].

In 1960s Warner and coworkers developed a model of HRV, which considered the release, binding and degradation of Norepinephrine $(N E)$, and Acetyl choline (Ach) respectively at the neuro effector junction [12]. The model does not study interaction between sympathetic and parasympathetic systems. The model developed by Dokos in 1996 was the first to address the kinetics outside the neuro effector junction [13]. A comprehensive model of SA node cell rate regulation is presented by Scepanovic et.al which incorporates second messenger kinetics also in addition to neurotransmitter kinetics [14]. Consequently the model has more parameters and hence computationally intensive. 


\section{Proposed model of ANS for Heart Rate Variability}

The proposed study is a mathematical model of neurotransmitter release, binding and its reuptake that reflect a change in HRV. The model describes the kinetics at Neuro effector Junction (NJ), Extra Junctional Space (EJS), and Extra Cellular Matrix (ECM).

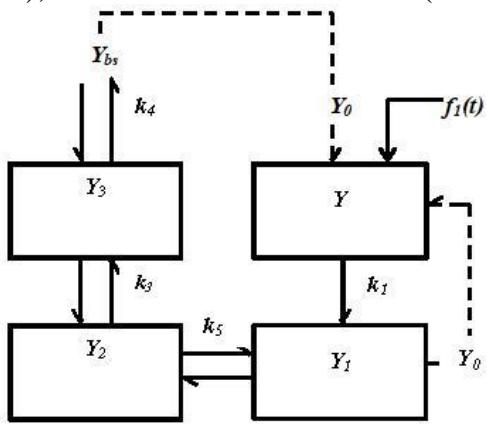

Figure 1(a). Diagram of the model at sympathetic site. Sinusoidal stimulation $f_{l}(t)$ causes release of Norepinephrine $(N E), Y$ is the number of $N E$ filled vesicles $Y_{1}, Y_{2}, Y_{3}$ are the concentration of $N E$ at Neuro effector Junction, Extra Junctional Space and Extra Cellular Matrix. Compartments shown in solid lines are modeled explicitly and dashed lines shows parameters which are not modeled explicitly since they are assumed to be constants

As shown in Fig 1 (a), at sympathetic site $Y$ is the number of $N E$ filled vesicles and $N_{\max N E}$ is the maximum number of vesicles per nerve ending. $Y_{1}, Y_{2}, Y_{3}$ are the concentration of $N E$ at NJ, EJS, and ECM respectively. Kinetics at sympathetic site is explained by the following equations.

$$
\begin{aligned}
& \frac{d Y(t)}{d t}=\frac{N_{\max N E}-Y(t)}{t a u_{N E}}+\frac{v_{2} Y_{1}(t)}{Q_{N E}}-k_{1} Y(t) f_{1}(t) \\
& \frac{d Y_{1}(t)}{d t}=\frac{Q_{N E}}{v_{2}} Y(t) k_{1} f_{1}(t)+k_{5}\left(Y_{2}(t)-Y_{1}(t)\right) \\
& \frac{d Y_{2}(t)}{d t}=k_{5}\left(Y_{1}(t)-Y_{2}(t)\right)+k_{3}\left(Y_{3}(t)-Y_{2}(t)\right) \\
& \frac{d Y_{3}(t)}{d t}=k_{3}\left(Y_{2}(t)-Y_{3}(t)\right)+k_{4}\left(Y_{b s}-Y_{3}(t)\right)
\end{aligned}
$$

$N(t)$ is the resultant signal produced at the sympathetic site in response to sympathetic stimulation

$$
N(t)=\frac{Y_{1}(t) v_{2}+Y_{2}(t) v_{3}}{V}
$$

$v_{l}, v 2, v 3, V$ are total volume of cholinergic $\mathrm{NJ}$, total volume of adrenergic NJ, volume of EJS and volume of cytosol inside SA node cell respectively. $Y_{b s}, Y_{0}, X_{b s}$, and $X_{0}$ are the concentration of $N E$ in the blood stream, $N E$ getting synthesized, Ach in the blood stream and Ach getting synthesized which are assumed to be constants. $k l=.01 / \mathrm{s} ; k 2=7800 / \mathrm{s} ; k 3=20 / \mathrm{s} ; k 4=.4 / \mathrm{s} ;$ and $k 5=8800 / \mathrm{s}$. The instantaneous neural stimulus at the sympathetic and parasympathetic sites are $f_{1}(t)$ and $f_{2}(t)$ respectively.

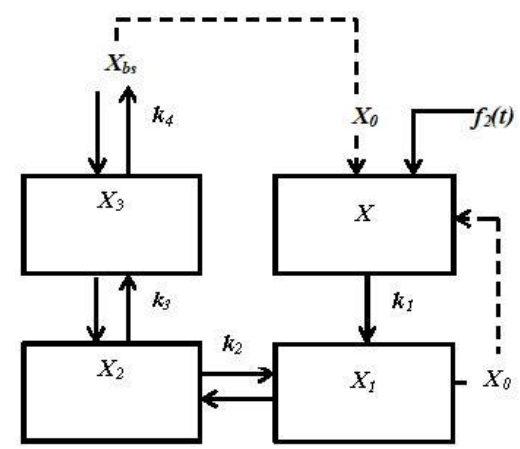

Figure 1(b).Diagram of the model at parasympathetic site. Sinusoidal stimulation $f_{2}(t)$ causes release of Ach, $X$ is the number of Ach filled vesicles $X_{1}, X_{2}, X_{3}$, are the concentration of Ach at Neuro effector Junction, Extra Junctional Space and Extra Cellular Matrix. Compartments shown in solid lines are modeled explicitly and dashed lines shows parameters which are not modeled explicitly since they are assumed to be constants

As shown in Fig 1(b), at vagal site, $X$ is the number of Ach filled vesicles. $N_{\max A c h}$ is the maximum number of vesicles filled with $A C h . X_{1}, X_{2}, X_{3}$, are the concentration of Ach at NJ, EJS, and ECM respectively. Reactions at the vagal site are represented by the following equations.

$$
\begin{aligned}
& \frac{d X(t)}{d t}=\frac{N_{\text {max Ach }}-X(t)}{t a u_{A c h}}+\frac{X_{1}(t) v_{1}}{Q_{A c h}}-k_{1} X(t) f_{2}(t) \\
& \frac{d X_{1}(t)}{d t}=\frac{Q_{A c h}}{v 1} k_{1} X(t) f_{2}(t)+k_{2}\left(X_{2}(t)-X_{1}(t)\right) \\
& \frac{d X_{2}(t)}{d t}=k_{2}\left(X_{1}(t)-X_{2}(t)\right)+k_{3}\left(X_{3}(t)-X_{2}(t)\right) \\
& \frac{d X_{3}(t)}{d t}=k_{3}\left(X_{2}(t)-X_{3}(t)\right)+k_{4}\left(X_{b s}-X_{3}(t)\right)
\end{aligned}
$$

$A(t)$ is the resultant signal at parasympathetic site in response to vagal stimulus.

$$
A(t)=\frac{X_{1}(t) v_{1}+X_{2}(t) v_{3}}{V}
$$

It has been shown that there is mutual excitation and inhibition between both the branches of ANS [15]. Interaction between both branches of ANS is modeled by modifying standard Hill's equation [14]. Resultant vagal signal is further modified as in Eqn (11).

$\frac{d V(t)}{d t}=\frac{A(t)-V(t)}{\operatorname{tau}_{x}}$

Inhibiting effect of vagal activity $V(t)$ on sympathetic activity $S(t)$ is simulated as in Eqn (12).

$$
\begin{aligned}
& S(t)=N(t)\left(1-r \frac{V^{l}(t)}{V^{l}(t)+K^{l}}\right) \\
& m(t)=S(t)-V(t)
\end{aligned}
$$

$m(t)$ represents the output of the proposed ANS model (neural signal), $m_{0}$ is a constant corresponding to the intrinsic heart rate when there is no stimulation. $m_{0}$ is fixed to be a positive dc value and $m_{0}+m(t)$ is strictly positive. When there is no stimulation, the observed heart 
rate is $72 \mathrm{bpm}$. Rate constants have significance in the dynamics of the system, values are adopted from [14].

\section{ANS model in combination with FHN model

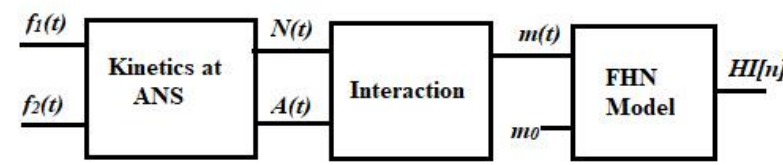

Figure 2. HRV model. Neural signal from the ANS model is fed to a FHN model to generate heart beat interval series.

The model developed for ANS kinetics has continuous time output hence we used a Fitzhugh-Nagumo (FHN) model as the next stage for generating discrete heart beat interval series. FHN is a reduced version of classical Hodgkin-Huxley $(\mathrm{H}-\mathrm{H})$ model. It models the activation and deactivation dynamics of a spiking neuron. The model is explained by two coupled nonlinear equations[16]

$$
\begin{aligned}
& \frac{d v}{d t}=a(-v(v-1)(v-b)-w) \\
& \frac{d w}{d t}=v-c w
\end{aligned}
$$

Where $v$ (fast variable) models the membrane potential, $\mathrm{w}$ (slow variable) models the recovery parameters of the membrane potential; $a$ and $c$ are scaling parameters. Moreover, $b$ is a variable with an unstable equilibrium that corresponds to the threshold between electrical silence and electrical firing. The parameters $a, b$, and $c$ are governed by internal mechanisms of the neuron. FHN model is modified as Eqn (16) for incorporating the dynamics of neurotransmitters, which includes a time varying threshold.

$$
b(t)=m_{0}+m(t)
$$

$H I[n]$ is the resulting heart beat interval series.

\section{Results and Discussions}

FHN model is a neuron model and action potential is not similar to that of cardiac action potential. By varying the parameters of the model it is made suitable to represent the heart beat series as in this study we are concerned with the heart beat interval and not ECG morphology. FHN output is illustrated in Figure 3 (i) and heart beat interval in Figure 3 (ii).

As shown in Fig 4, for a sympathetic input $f_{1}(t)=$ $\sin (2 \pi f t)$; and vagal input $f_{2}(t)=0$; the autonomic neural signal (ii) is almost in phase with the input stimulus (i), oscillates at the same frequency as that of the input and its value is always above the fixed dc level corresponding to $m_{0}$. Corresponding HRV signal (iii) is out of phase with stimulus. Range of regulation is in between 140 beats per minute (bpm) and 72bpm.
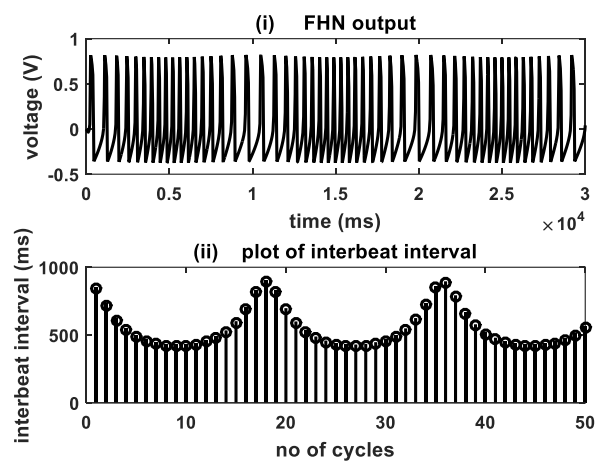

Figure 3 (i) FHN model output (ii) corresponding variation of inter beat interval for a sinusoidal input of 0.1 $\mathrm{Hz}$ applied at the sympathetic input of the model.
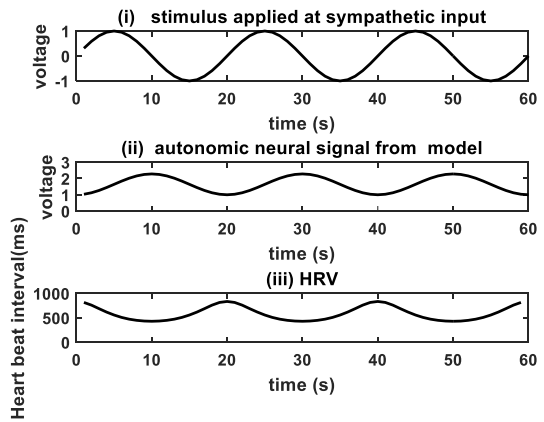

Figure 4 (i) Input stimuli, (ii) autonomic neural signal, (iii) HRV respectively for sympathetic input $f_{1}(t)=$ $\sin (2 \pi f t) ; f_{2}(t)=0$; where $f=.05 \mathrm{~Hz}$
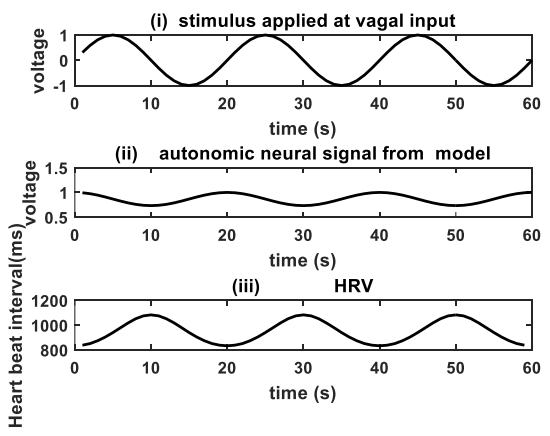

Figure 5(i) Input stimulus, (ii) Autonomic neural signal, (iii) HRV respectively for a vagal input $f_{2}(t)=$ $\sin (2 \pi f t) ; f_{1}(t)=0$; where $f=.05 \mathrm{~Hz}$

As in Figure 5, for a vagal input $f_{2}(t)=$ $\sin (2 \pi f t) ; f_{1}(t)=0$; the autonomic neural signal (ii) is almost out of phase with the input (i), oscillates at the same frequency of the input below the fixed dc level. Corresponding HRV signal (iii) is in phase with stimulus and the range of regulation is between $72 \mathrm{bpm}$ and 55 bpm. The range of regulation observed in both experiments is close to normal physiologic range. 
ECG data were recorded from a group of 50 healthy individuals having balanced sympathetic and parasympathetic activities and analyzed using KUBIOS HRV tool kit for typical HRV parameters [10]. HRV signal was synthesized by the model for the same autonomic state and analyzed using the same method.
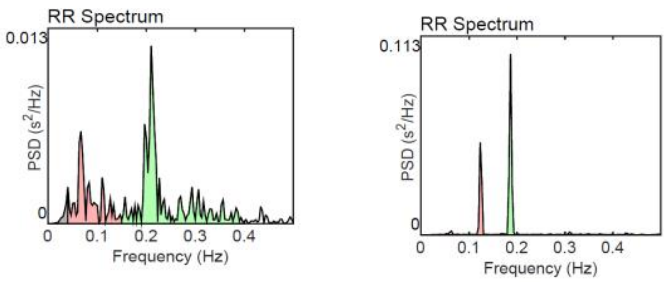

Figure 6 Power spectrum (a) Physiological data

(b) Model synthesized data

As illustrated in Figure 6 two peaks are observed in (b) similar to (a). The first peak reflects combined activity of sympathetic and parasympathetic system while the second peak corresponds to respiratory modulation of heart rate.
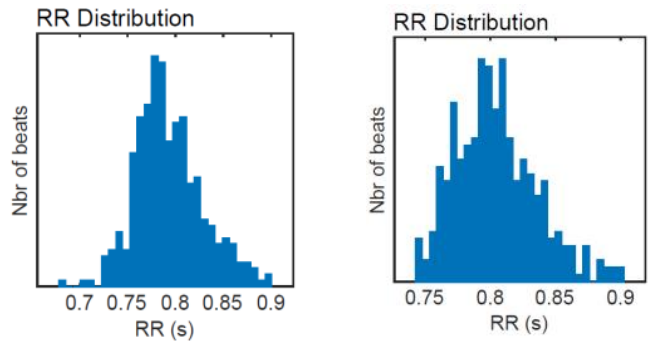

Figure 7 Distribution of interbeat interval. (a) Physiologic HRV data (b) Model synthesized HRV data

The model generated R-R interval distribution is almost in the same range as that of the physiologic data.

\section{Conclusion}

The developed ANS model reproduced HRV when used in combination with a FHN model, in the physiologic range. Heart rate regulation range, distribution of inter beat interval, and power spectrum observed for model synthesized HRV data is close to physiologic HRV data.

\section{References}

[1] J. E. Hall, Guyton and Hall textbook of Medical Physiology e-Book: Elsevier Health Sciences, 2010.

[2] C. Giessler, S. Dhein, K. Pönicke, and O.-E. Brodde, "Muscarinic receptors in the failing human heart," European Journal of Pharmacology, vol. 375, pp. 197-202, 1999.

[3] T. Kishi, "Heart failure as an autonomic nervous system dysfunction," Journal of Cardiology, vol. 59, pp. 117-122, 2012.
[4] A. H. Kemp, D. S. Quintana, M. A. Gray, K. L. Felmingham, K. Brown, and J. M. Gatt, "Impact of depression and antidepressant treatment on heart rate variability: a review and meta-analysis," Biological Psychiatry, vol. 67, pp. 1067-1074, 2010.

[5] J. M. Montaquila, B. J. Trachik, and J. S. Bedwell, "Heart rate variability and vagal tone in schizophrenia: a review," Journal of Psychiatric Research, vol. 69, pp. 57-66, 2015.

[6] E. Vanninen, A. Tuunainen, M. Kansanen, M. Uusitupa, and E. Länsimies, "Cardiac sympathovagal balance during sleep apnea episodes," Clinical Physiology, vol. 16, pp. 209-216, 1996.

[7] M. Karas, P. Larochelle, R. A. LeBlanc, B. Dubé, R. Nadeau, and J. d. Champlain, "Attenuation of autonomic nervous system functions in hypertensive patients at rest and during orthostatic stimulation," The Journal of Clinical Hypertension, vol. 10, pp. 97 104, 2008.

[8] H. Kudat, V. Akkaya, A. Sozen, S. Salman, S. Demirel, M. Ozcan, D. Atilgan, M. Yilmaz, and O. Guven, "Heart rate variability in diabetes patients," Journal of International Medical Research, vol. 34, pp. 291-296, 2006.

[9] F. d. r. Roche, J.-M. Gaspoz, I. Court-Fortune, P. Minini, V. Pichot, D. Duverney, F. d. r. Costes, J.-R. Lacour, and J.-C. Barthélémy, "Screening of obstructive sleep apnea syndrome by heart rate variability analysis," Circulation Research, vol. 100, pp. 1411-1415, 1999.

[10] M. P. Tarvainen, J.-P. Niskanen, J. Lipponen, P. Ranta-Aho, and P. Karjalainen, "Kubios HRV-a software for advanced heart rate variability analysis," in 4th European conference of the International federation for Medical and Biological Engineering, 2009, pp. 1022-1025.

[11] F. Shaffer, R. McCraty, and C. L. Zerr, "A healthy heart is not a metronome: an integrative review of the heart's anatomy and heart rate variability," Frontiers in Psychology, vol. 5, p. 1040, 2014.

[12] H. R. Warner and A. Cox, "A mathematical model of heart rate control by sympathetic and vagus efferent information," Journal of Applied Physiology, vol. 17, pp. 349-355, 1962.

[13] S. Dokos, B. Celler, and N. Lovell, "Vagal control of sinoatrial rhythm: a mathematical model," Journal of Theoretical Biology, vol. 182, pp. 21-44, 1996.

[14] D. Śćepanović, "A model of sinoatrial node cell regulation by the autonomic nervous system," Massachusetts Institute of Technology, 2011.

[15] M. N. Levy, "Brief reviews: sympatheticparasympathetic interactions in the heart," Circulation Research, vol. 29, pp. 437-445, 1971.

[16] R. FitzHugh, "Impulses and physiological states in theoretical models of nerve membrane," Biophysical Journal, vol. 1, pp. 445-466, 1961.

Address for correspondence:

Sajitha S.

Model Engineering College,Kochi-682 021, India. sajitha@mec.ac.in 